\title{
Zur Gliederung der Holstein- und Saalezeit im östlichen Harzvorland $\left.{ }^{1}\right)$
}

\author{
Von Ralf Ruske, Halle (Saale) \\ Mit 4 Abbildungen und 1 Tabelle im Text
}

\begin{abstract}
$\mathrm{Zus}$ a mmen f as ung. Es wird ein Uberblick über die Ergebnisse von Untersuchungen im Pleistozän des östlichen Harzvorlandes gegeben. Es wurde eine Terrasse gefunden, die während eines Kälteeinbruchs innerhalb der Holsteinzeit aufgeschottert wurde.

Die frühsaalezeitlichen Hauptterrassen der Flüsse zeigen zwei Sedimentationszyklen. Die ihnen zeitlich entsprechenden Lößserien weisen eine gleichartige Gliederung auf. Zwischen beiden Zyklen liegt eine interstadiale Bodenbildung. Der Hauptvorstoß des Saaleeises endete im östlichen Harzvorland. $\mathrm{Er}$ läßt sich lokal durch kleine Oszillationen des Eisrandes weiter untergliedern.

Wartheglazialen Alters sind eine Terrasse und Löß. Zwischen der Saale- und der Warthevereisung liegt ein wärmerer Zeitabschnitt, in dem ein Bodenkomplex entstanden ist.

Es wird vorgeschlagen, die Begriffe Eiszeit und Warmzeit in den Pleistozängliederungen zu vermeiden und nur von Saalezeit, Holsteinzeit usw. zu sprechen.
\end{abstract}

$\mathrm{Summary}$. A review is given on the results of researches which were undertaken in the Pleistocene of the eastern foot-hills of the Harz. A terrace could be detected where the gravels have been accumulated during a cold period in the Holstein stage.

The main terraces of the rivers, corresponding in age to the early Saale stage, indicate two sedimentary cycles. Two sequences of loess can be correlated with the sedimentary cycles of the rivers; the loess is of equal constitution. An interstadial soil-formation can be observed in between both cycles. Maximum ice advance of the Saale glaciation came to an end at the eastern foot-hills of the Harz. This zone locally can be subdivided by minor movements of the ice sheet.

A terrace and loess can be correlated with the Warthe glaciation. Inbetween both the Saale and the Warthe glaciation a temperate period caused formation of soils.

The proposal is made to avoid terms like glacial and interglacial, when dealing with Pleistocene stratigraphy. In such a case only terms like Holstein stage, Saale stage etc. should be used.

\section{Vorbemerkungen}

Die erste detaillierte Gliederung von Schichten der Holstein- und Saalezeit im östlichen Harzvorland nahmen L. Siegert \& W. WeISSERmel (1911) vor. Sie untersuchten das Pleistozän im Bereich der Saale zwischen Halle und Weißenfels und fanden zwei zwischen der Elster- und Saalevereisung entstandene Flußterrassen und eine dreigegliederte Grundmoräne der Saalevereisung.

R. Grahmann (1925) wies in Nordwestsachsen gleichfalls drei saalezeitliche Eisvorstöße nach. Er fand aber nur eine Terrasse, die er der Saalezeit zuordnete. In den späteren Jahren wurden durch den Bergbau und andere Industrien eine Vielzahl von Bohraufschlüssen und Gruben geschaffen, deren systematische Bearbeitung vor allem durch die Institutionen der früheren Staatlichen Geologischen Kommission und das GeologischPaläontologische Institut der Universität Halle durchgeführt wurde. Ein Überblick über die bisherigen Ergebnisse wird nachfolgend gegeben.

Der Raum, der im Rahmen dieses Aufsatzes behandelt wird, endet im Süden an der Finne, im Westen am Harz, im Norden an der Wipper und im Osten an der Mulde.

1) Vortrag, gehalten auf der DEUQUA-Tagung im Oktober 1964 in Lüneburg, ergänzt; zugleich Mitteilung aus dem VEB Geologische Erkundung West. 


\section{Die Abfolge der holstein- und saalezeitlichen Sedimente}

Die Grenze der Maximalausdehnung des Saaleeises verläuft von NW nach SE durch das östliche Harzvorland. Südlich des ehemaligen Eisrandes treten in großer Verbreitung äolische und fluviatile mittelpleistozäne Sedimente auf und nördlich vorwiegend glaziäre und fluviatile. Im jüngeren Teil der Saalezeit (Warthevereisung) lag die Vereisungsgrenze bereits außerhalb des östlichen Harzvorlandes nördlich der Elbe und Ohre, so daß nur Sedimente des Periglazials vorhanden sind.

2.1 Die holstein-und saalezeitlichen fossilen Böden und Lösse

Lösse sind vornehmlich im Gebiet zwischen der Saale, Unstrut und dem Harz verbreitet. Sie enthalten in verschiedenen Aufschlüssen ältere fossile Böden, deren stratigraphische Einstufung mit Hilfe von Flußterrassen oder glazigenen Sedimenten vorgenommen werden konnte.

Einen holsteinzeitlichen fossilen Bodenkomplex, der unter saalezeitlichem Löß lagert, beschrieben R. RUSKE \& M. WüNSCHE $(1961,1964)$ aus der Ziegeleigrube Freyburg/U. Dieser "Freyburger Bodenkomplex" besteht aus einer basalen Parabraunerde und einer darüberliegenden Humuszone. R. Kunert \& M. Altermann (1965) fanden holsteinzeitliche Schotterlehme im Bereich des Wippertales am östlichen Harzrand.

Die saalezeitliche Sedimentation wird in den Lößprofilen mit einer Fließerde eingeleitet, die z. T. humos ist und verlagertes Bodenmaterial enthält. Sie geht in Schwemmlöß

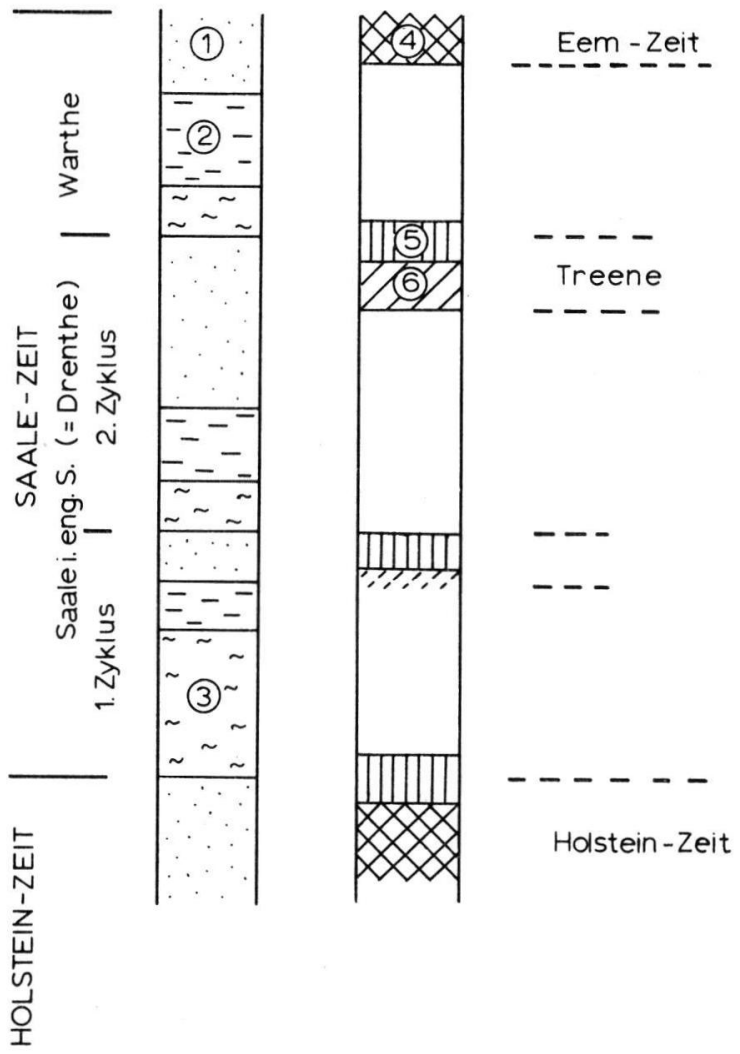

Abb. 1. Holstein- und saalezeitliche Lösse und fossile Böden im östlichen Harzvorland.

$1=$ Fluglöß; $2=$ Schwemmlöß; $3=$ Fließerde; $4=$ Parabraunerde; $5=$ Humuszone (zumeist Tschernosem); 6 = Braunerde. 
über, aus dem schließlich Fluglöß hervorgeht. Der Fluglöß wurde in seinen oberen Lagen von einem degradierten Tschernosem überprägt, der ein frühsaalezeitliches Interstadial anzeigt. In seinem Hangenden schließen sich Fließerden, Schwemmlöß und der „Hauptlöß“ der Saalezeit an, der sich im Raum der unteren Unstrut mit dem zum Hauptvorstoß der Saalevereisung gehörenden Bruckdorfer Beckenton verzahnt.

Über dem „Hauptlöß“ der Saalezeit folgt ein fossiler Bodenkomplex, den im unteren Unstruttal R. Ruske \& M. Wünsche (1961) als Rudelsburger Bodenkomplex bezeichnet haben. Er zeigt im Aufschluß Bad Kösen Merkmale eines Auenbodens mit einer Humuszone, bei Freyburg ist er als Braunerde mit Humuszone und bei Langenbogen westlich Halle als Naßboden mit Humuszone (R. Kunert \& M. Altermann 1965) ausgebildet. In keinem Aufschluß konnte in dem fossilen Bodenkomplex eine Tonverlagerung beobachtet werden, die auf ein Parabraunerdestadium hinweist. Über ihm folgen Fließerden und Löß, die warthezeitlichen Alters sind. Sie wurden z. T. durch die eemzeitliche Bodenbildung überprägt.

Die eben beschriebene Löß-Bodenfolge (Abb. 1) zeigt eine Teilung der Saalezeit in kalte und relativ warme Abschnitte, die durch Lösse bzw. fossile Böden charakterisiert werden. Ein erster saalezeitlicher relativ '„warmer“ Abschnitt liegt vor der Inlandvereisung und ein zweiter zwischen der Saale- und der Warthevereisung.

2.2 Die holstein- und saalezeitlichen Akkumulationsterrassen der Flüs se

Die Entwicklungsgeschichte der mitteldeutschen Flüsse konnte in den letzten Jahren durch die Anwendung quantitativer Untersuchungsmethoden (z. B. Schwermineralanalyse, quantitative Schotteranalyse) und die Auswertung zahlreicher Bohrproben aufgehellt werden. Es wurden neue Flußläufe bzw. die Fortsetzungen bekannter Flüsse nach Norden gefunden und ihre Terrassen stratigraphisch fixiert. Wesentliche Ergebnisse konnten dort erzielt werden, wo die Flüsse aus dem weiteren Mittelgebirgsbereich in das norddeutsche Flachland austraten und durch zahlreiche spätere Laufverlegungen alte Terrassen erhalten geblieben sind.

Die ältesten Flußschotter, die jünger als die Elstervereisung sind, fand W. KNOTH (1964) nördlich Halle in der Umgebung von Zörbig. Sie gehören einem spätelsterzeitlichen bis frühholsteinzeitlichen(?) Saalelauf an, in den von Osten kommend die Mulde gemündet ist. Die Kiese zeichnen sich an der Basis durch einen hohen Gehalt an nordischem Material aus und gehen nach oben in typische Saalekiese über. Die Terrasse konnte in anderen Flußgebieten des östlichen Harzvorlandes bisher nicht gefunden werden.

Über dem holsteinzeitlichen Interglazial von Edderitz folgt eine Terrasse, die holsteinzeitlichen Alters ist (Abb.2). Sie ist zwischen Halle und Dessau weit verbreitet, enthält syngenetische Frostbodenstrukturen und wird als Edderitze r T e r r a s e bezeichnet (R. Ruske 1964; W. KNOTH 1964). Südlich Halle liegt sie zwischen der frühelsterzeitlichen und der frühsaalezeitlichen Aufschotterung. Die Kiese sind in ihrem oberen Teil oft stark verwittert. M. Altermann (in W. KNOth 1964) bezeichnet den Verwitterungshorizont als "braunlehmartigen, gelblichroten Interglazialboden“.

Die nächstjüngere fluviatile Aufschotterung wird im östlichen Harzvorland als Hauptterrasse bezeichnet (L. Siegert \& W. Weissermel 1911), weil dieser Schotterkörper am weitesten verbreitet und am besten erhalten ist. Im Gebiet der Unstrut wurde er z. B. durch R. Lehmann (1922) und H. Kugler (1961), im Bereich der unteren Saale durch L. Siegert \& W. Weissermel (1911), V. Toepfer (1933), W. Schulz (1962), R. Ruske (1962) und W. KNOтн (1964) und im Raum der Elster und Mulde durch R. Grahmann (1925) und L. Eissmann (1962) untersucht. Der Schotterkörper enthält zwei Sedimentationszyklen, die mit Kiesen beginnen und mit feinem Sand bis Schluff enden. An ihrer Oberkante zeigen sie z. T. solifluidale Umlagerungen und Kryoturbationen (ein Teil dieser kryogen über- 
formten Sedimente wurde in der älteren Literatur als Grundmoräne bezeichnet!). Während im Muldegebiet der obere Zyklus der Hauptterrasse mächtiger ist (L. Eissmann 1962), dominiert im Bereich der Saale der untere. Nach dem ersten Zyklus verließen die Saale und Mulde nördlich Halle an mehreren Stellen ihr Bett und schotterten den Köthener SaaleMuldelauf auf, der sich nach etwa $25 \mathrm{~km}$ Erstreckung wieder mit der Saale vereinigt hat. Die Hauptterrassen werden größtenteils von den glaziären Sedimenten der Saalevereisung bedeckt.

Nach der Saalevereisung wurden im östlichen Harzvorland zwei morphologisch in Erscheinung tretende Terrassen aufgeschottert. Die ältere liegt etwa $6-8 \mathrm{~m}$ über der heutigen Flußaue und trägt an verschiedenen Stellen Reste der eemzeitlichen Bodenbildung (Bt-Horizont einer Parabraunerde). Sie ist jünger als die Saalevereisung und älter als die Eemzeit und wird mit der Warthevereisung parallelisiert.

Die warthezeitliche Terrasse ist schlecht aufgeschlossen, so daß keine Auskunft darüber gegeben werden kann, ob der Schotterkörper einheitlich ist oder Teile von ihm in das Spätglazial der Saalevereisung gestellt werden müssen.

Die jüngste, deutlich sichtbare Aufschotterung ist die weichselzeitliche Niederterrasse.

2.3 Die glaziären saalezeitlichen Bildungen und die Zahl der E is vorstöße

Aus dem östlichen Harzvorland haben L. Siegert \& W. Weissermel (1911), R. Grahmann (1925), K. Groth (1961), W. Schulz (1961), R. Ruske (1961) und andere mehrere Vorstöße des Saaleeises beschrieben, die zumeist als Basal-, Haupt- und Deckvorstoß bezeichnet wurden. $\mathrm{Zu}$ ihnen gehören Bändertone, Grundmoränen und Toteissande. In ihrer vollen Ausbildung konnte diese Schichtenfolge nur in der Umgebung von Halle beobachtet werden. In den anderen Gebieten ist zumeist nur die Grundmoräne des Haupt-

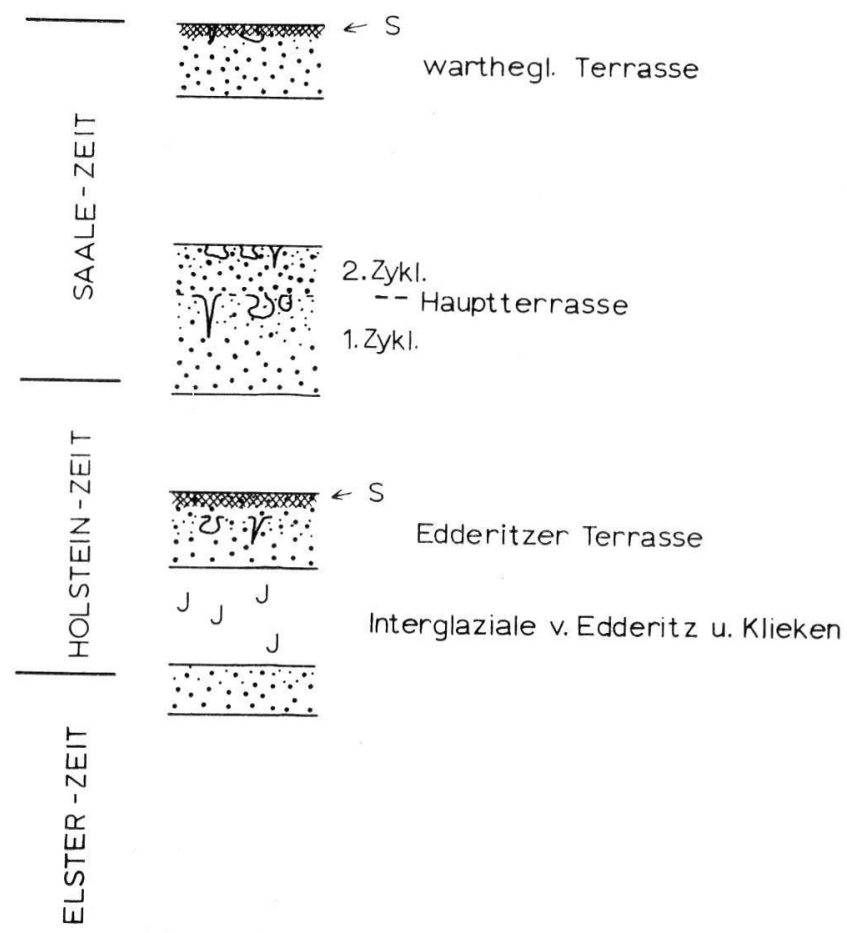

Abb. 2. Holstein- und saalezeitliche fluviatile Aufschotterungen östlich des Harzes. S=Schotterlehm. 
vorstoßes vorhanden. Der Basalvorstoß des Saaleeises ist nach Auffassung des Verfassers nur eine kleine Oszillation des Hauptvorstoßes, die auf die nähere Umgebung von Halle beschränkt ist. Er besitzt keine stratigraphische Bedeutung für eine großräumige Gliederung der Saalevereisung. Der Hauptvorstoß des Saaleeises ist der am weitesten nach Süden

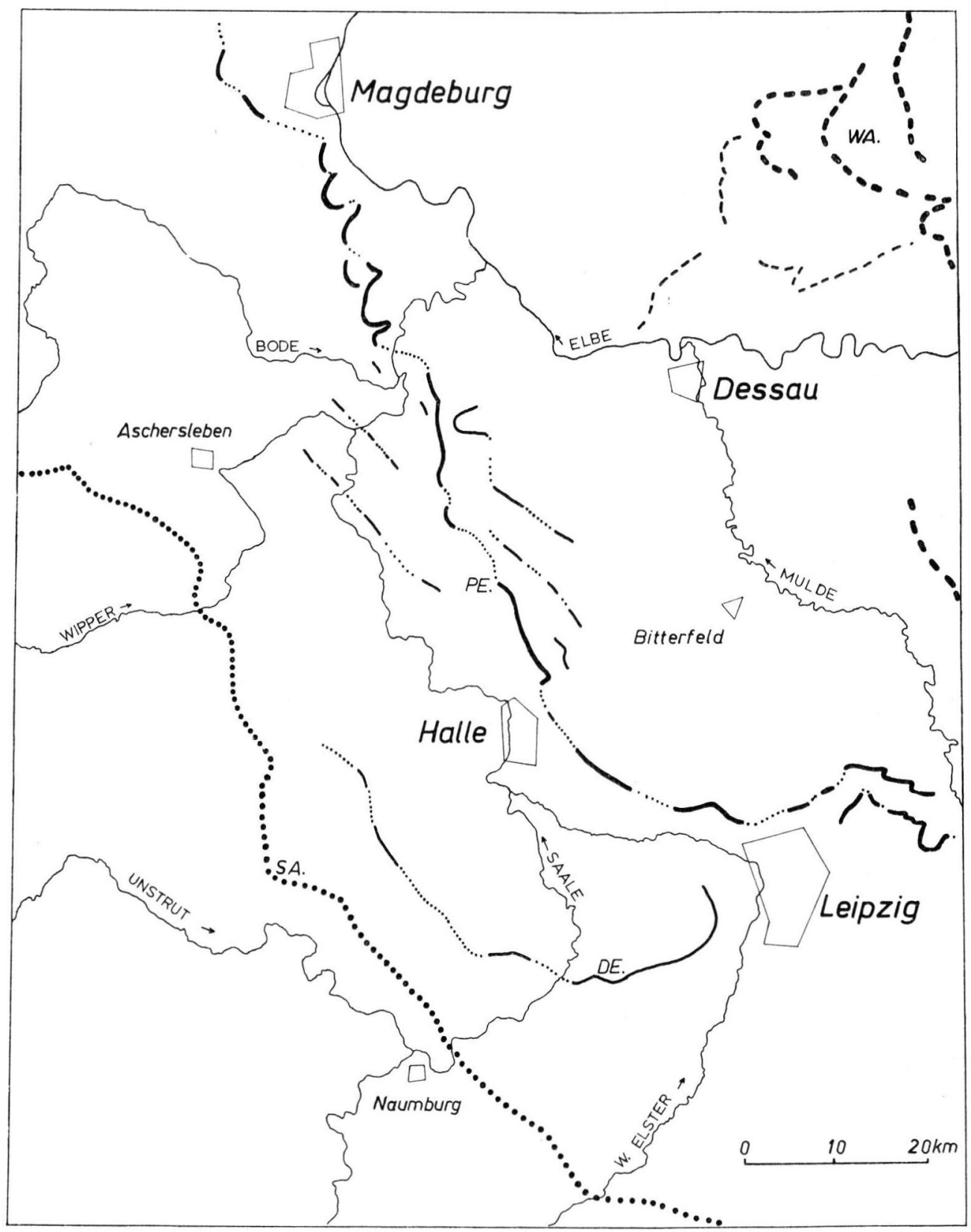

Abb. 3. Saalezeitliche Eisrandlagen im östlichen Harzvorland.

SA. = Maximalausdehnung des Saaleeises; DE. $=$ Dehlitzer Randlage; PE. = Petersberger Eisrandlage; WA. = warthezeitliche Endmoränen. 
reichende. Seine Maximalausdehnung ist z. T. gut bekannt (Abb. 3). Zu ihm gehören mehrere Staffeln eisrandparalleler Ablagerungen (Endmoränen, Sander usw.), die entweder dem vorrückenden Eis oder dem sich anschließenden Eisabbau zugeordnet werden. Nach dem Hauptvorstoß stieß das Saaleeis nochmals bis in die Umgebung von Halle vor. Die Randlage dieses vom Verfasser (1964) „Petersberger Vorstoß“ genannten Eisvorstoßes kann nach Norden bis in den Raum westlich Magdeburg verfolgt werden.

Einem jüngeren Eisvorstoß gehören die nördlich der Elbe gelegenen Endmoränen des Flämings und die zwischen Elbe und Mulde befindlichen endmoränenartigen Ablagerungen in der Dübener Heide an. Sie werden der Warthevereisung zugeordnet.

Die wichtigsten Glieder in den glaziären Ablagerungen der Saalezeit sind die Hauptgrundmoräne der Saalevereisung und die Warthemoräne (Abb. 4). Sie bestimmen die stratigraphische Einstufung der hangenden und liegenden Sedimente.

\section{Der Ablauf des Geschehens zwischen dem Ende der Elster- und Saalezeit im östlichen Harzvorland}

Die Beschreibung des Ablaufes basiert auf den Ergebnissen der Lößforschung, der Terrassenstratigraphie und der Untersuchung der Vereisungen. Sie gibt den jetzigen Stand der Kenntnis wieder (Tab. 1).

An das Ende der Elstereiszeit sind Flußschotter zu stellen, die lokal nördlich Halle auftreten und welche in ihren unteren Lagen noch Anklänge an glazifluviatile Kiese zeigen. Sie reichen möglicherweise bis in die Holsteinzeit hinein.

In den ersten warmzeitlichen Abschnitt der Holsteinzeit gehören die Interglaziale von Edderitz bei Köthen und Klieken bei Dessau. Beide liegen im Bereich elsterzeitlicher Entwässerungsrinnen, zeigen den Übergang vom Glazial- zum Interglazialklima, enthalten das interglaziale Klimaoptimum mit Eichenmischwald und enden mit einer kühlen Phase. Das Interglazial von Edderitz, das zuletzt W. KNOTH \& G. LenK (1962) untersucht haben, liegt im Bereich der Terrassen des Saalesystems.

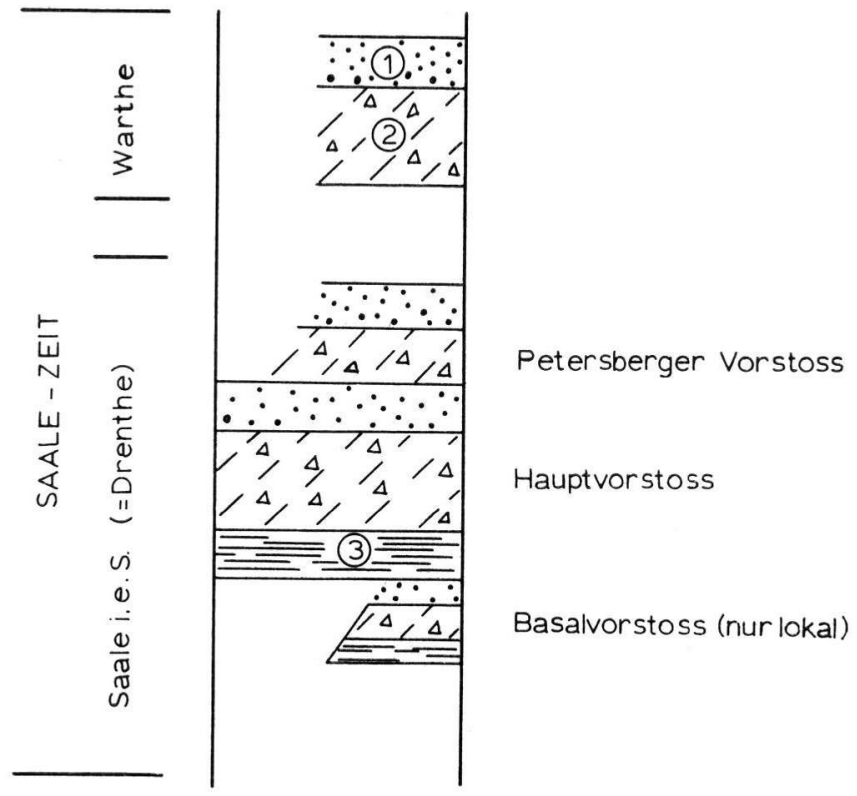

Abb. 4. Gliederung der saalezeitlichen glaziären Ablagerungen.

$1=$ glazifluviatile Sande und Kiese; $2=$ Grundmoräne; $3=$ Staubeckensedimente (zumeist Bänderton). 
Die Klimaverschlechterung, die im oberen Teil der beiden Interglaziale angezeigt wird, führte zur Aufschotterung der Edderitzer Terrasse. W. Ккотн (1964) nennt diesen kalten Abschnitt „Fuhnekälteschwankung “.

Nach der Sedimentation der Edderitzer Terrasse trat eine Klimaverbesserung ein und es folgte der zweite warmzeitliche Abschnitt der Holsteinzeit. Relikte aus diesem Zeitraum sind Schotterlehme, die in den basalen Teilen der Hauptterrassen enthaltenen Faunen mit Corbicula fluminalis und wahrscheinlich auch der Freyburger Bodenkomplex. Die basale Parabraunerde dieses fossilen Bodenkomplexes ist auf Löß entwickelt, der seiner Höhenlage nach erst nach der Elstervereisung während eines kalten Abschnittes der Holsteinzeit angeweht wurde (R. Ruske \& M. WüNSCHE 1964).

Während des ersten warmen Abschnittes und der Kälteschwankung der Holsteinzeit vereinigten sich die Mulde und Saale bereits $15 \mathrm{~km}$ nordöstlich Halle zu einem einheitlichen Flußlauf. Im zweiten warmen Abschnitt der Holsteinzeit verlegten die Mulde und

\section{Tabelle 1}

Gliederung der Holstein- und Saalezeit im östlichen Harzvorland

\begin{tabular}{|c|c|c|}
\hline \multicolumn{2}{|c|}{ 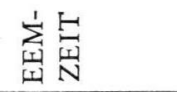 } & fossile Böden, z. B. Parabraunerde des Naumburger Bodenkomplexes \\
\hline \multirow{5}{*}{ 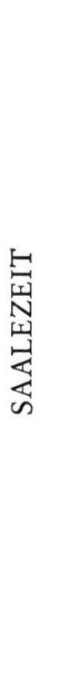 } & 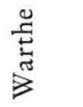 & $\begin{array}{l}\text { Ablagerungen des Eisvorstoßes; Löß und Lößderivate; Flußschotter } \\
\text { (?) Humuszone des Rudelsburger Bodenkomplexes }\end{array}$ \\
\hline & 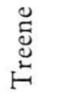 & fossile Böden, z. B. Rudelsburger Bodenkomplex \\
\hline & \multirow[t]{3}{*}{ 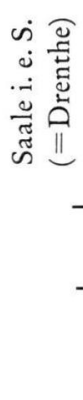 } & $\begin{array}{l}\text { Ablagerungen des Petersberger Vorstoßes der Saalevereisung } \\
\text { Ablagerungen des Hauptvorstoßes des Saaleeises einschließlich des Basal- } \\
\text { vorstoßes; Löß und Lößderivate } \\
\text { Zweiter Sedimentationszyklus der Hauptterrassen }\end{array}$ \\
\hline & & fossiler Boden (degr. Tschernosem) \\
\hline & & $\begin{array}{l}\text { Löß und Lößderivate. Erster Sedimentationszyklus der Hauptterrassen } \\
\text { (?) Humuszone des Freyburger Bodenkomplexes }\end{array}$ \\
\hline \multirow{3}{*}{ 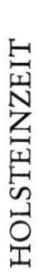 } & & $\begin{array}{l}\text { fossile Böden: Parabraunerde des Freyburger Bodenkomplexes und Schotter- } \\
\text { lehme }\end{array}$ \\
\hline & $\underset{\Xi}{\stackrel{\Xi}{\Xi}}$ & Edderitzer Terrasse; (?) Löß \\
\hline & & Interglaziale von Edderitz und Klieken \\
\hline \multicolumn{2}{|c|}{ 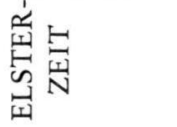 } & $\begin{array}{l}\text { Ablagerungen der Eisvorstöße } \\
\text { Flußschotter }\end{array}$ \\
\hline
\end{tabular}


Saale ihren Lauf und die Vereinigung erfolgte $50 \mathrm{~km}$ nördlich Halle im Bereich des heutigen Elbtales. Demzufolge ist es möglich, die älteren und jüngeren Terrassen auf Grund ihrer Geröllführung voneinander zu trennen.

Am Ende der Holsteinzeit setzte die Aufschotterung der Hauptterrassen ein. In den Lößprofilen liegt die Grenze zur Saalezeit vermutlich in der Humuszone des Freyburger Bodenkomplexes. Die Lößprofile zeigen vor dem Vorstoß des Saaleeises zwei Sedimentationszyklen, die mit einer Solifluktionsphase beginnen und mit kaltaridem Löß enden. Es ist wahrscheinlich, daß die Zweigliederung des saalezeitlichen Lösses mit der Teilung der Hauptterrasse in zwei Sedimentationszyklen in direktem Zusammenhang steht. Zwischen beiden Zyklen liegt ein wärmerer Abschnitt, in dem sich auf dem Löß ein degradierter Tschernosem entwickelte.

Der Vorstoß des saalezeitlichen Inlandeises beendete die Aufschotterung der Hauptterrassen. Nur die äolische Sedimentation setzte sich fort, so daß sich an der Vereisungsgrenze „Hauptsaalelöß“ und Bändertone verzahnen. Die Dreigliederung der Saalevereisung im engeren Sinne in Basal-, Haupt- und Deckvorstoß ist nur örtlich vorhanden. Sie konnte in den Lößprofilen nicht nachgewiesen werden.

Zwischen den zwei großen kalten Abschnitten der Saalezeit, die durch die Saale- und die Warthevereisung repräsentiert werden, lag ein wärmerer Zeitraum, in dem sich ein fossiler Bodenkomplex ausbildete. Er konnte an mehreren Stellen sicher nachgewiesen werden. Teils liegt der Boden über Sedimenten der Saalevereisung, teils über der Hauptterrasse und teils in Entwässerungsrinnen der Saalevereisung. Er wird von Warthelöß und der eemzeitlichen Bodenbildung überlagert. In keinem Aufschluß ist der Boden als Parabraunerde ausgebildet, auch konnte in keinem Profil nachgewiesen werden, daß der Boden vorher ein Parabraunerdestadium durchlaufen hat. Damit unterscheidet er sich wesentlich von den eem- bzw. holsteinzeitlichen Böden dieses Raumes, die als Parabraunerden bzw. Parabraunerde-Pseudogleye vorliegen.

Der zweite große Kälteabschnitt der Saalezeit, dem die Warthevereisung angehört, wird im östlichen Harzvorland nur durch Sedimente des Periglazials vertreten. Es ist eine Flußterrasse bekannt, und in den Lößprofilen kann ein Sedimentationszyklus beobachtet werden, der mit Fließerde beginnt und mit Löß endet. Eine weitere Untergliederung dieses Zeitraumes kann nicht vorgenommen werden.

Die saalezeitlichen Ablagerungen tragen an zahlreichen Stellen Relikte der eemzeitlichen Bodenbildung, die für die stratigraphische Einstufung der Sedimente wichtig sind.

\section{Zu den Begriffen Holsteinzeit und Saalezeit}

Die bisherigen Untersuchungen im Pleistozän des östlichen Harzvorlandes haben gezeigt, daß die in der Literatur zumeist als Holstein-Interglazial (-Warmzeit) oder SaaleEiszeit (-Kaltzeit) bezeichneten Zeiträume aus einem Wechsel von kalten und warmen Zeitabschnitten bestehen. In der Holsteinzeit dominieren die wärmeren und in der Saalezeit die kälteren. Holsteinzeitlich ist aber auch eine Schotterterrasse, die unter periglazialen Klimaverhältnissen entstanden ist. Sie müßte, den bisherigen Bezeichnungen folgend, holsteininterglaziale Terrasse genannt werden. $\mathrm{Da}$ es jedoch nicht immer möglich ist, den Charakter der Aufschotterung eingehend zu erläutern, könnte dies zu falschen Schlußfolgerungen führen. Ein Beispiel mit entgegengesetztem Vorzeichen ist der nach der Saalevereisung und vor der Warthevereisung entstandene Rudelsburger Bodenkomplex, der auf eine deutliche Erwärmung innerhalb der Saalezeit hinweist.

Um das Holstein-Interglazial und die Saale-Eiszeit von einer strengen klimatischen Festlegung zu entlasten, wird vorgeschlagen, die Zusätze „Interglazial“ bzw. „Eiszeit“ zu streichen und durch „Zeit“ zu ersetzen - Holsteinzeit und Saalezeit. Angewandt auf die 
gesamte Gliederung des Pleistozäns würde dies bedeuten, daß von Weichselzeit, Eeemzeit, Saalezeit usw. gesprochen werden müßte. Diese Bezeichnungen lassen die Möglichkeit offen, die Namen auch dann beizubehalten, wenn es sich herausstellen sollte, daß z. B. die Elsterzeit durch eine Warmzeit untergliedert werden muß oder die Cromerzeit durch eine Kaltzeit.

\section{Schriftenverzeichnis}

DiezemanN, W.: Beiträge zur hydrologischen Entwicklung des Saale-Salzke-Gebietes vom Elster/ Saale-Interglazial zum Saale/Weichsel-Interglazial in der Umgebung von Halle (Saale). Jb. Hall. Verb. 17, 11-56, Halle 1939.

Eissmann, L.: Riß- und mindelglaziale Eisrandlagen und Flußterrassen zwischen Mulde und Pleiße im Leipziger Raume. - Exkursionsführer zur Herbsttagung der Geol. Gesellsch. i. d. DDR, 31-49, Berlin 1962.

Grahmann, R.: Diluvium und Pliozän in Nordwestsachsen. - Abh. sächs. Akad. Wiss., math.-nat. Kl., 39, Nr. IV, Leipzig 1925.

Grotн, K.: Beitrag zur Gliederung des Saaleglazials bei Halle (Saale) und im Mansfelder Seekreis. Geologie 10, 169-184, Berlin 1961.

KNотн, W. \& G. LenK: Das Pleistozänprofil des ehemaligen Tagebaues Edderitz im Kreis Köthen. Geologie 11, 1149-1163, Berlin 1962.

KNOтH, W.: Zur Kenntnis der pleistozänen Mittelterrassen der Saale und Mulde nördlich von Halle. - Geologie 13, 598-616, Berlin 1964.

Kugler, H.: Bemerkungen zur Gliederung und Altersstellung der pleistozänen Flußterrassen der Unstrut. - Geogr. Ber. 20/21, 183-196 (1961).

Kunert, R. \& M. Altermann: Das Pleistozän zwischen Saale und Wipper. - Geologie 15, Berlin 1965.

Lehmann, R.: Das Diluvium des unteren Unstruttales von Sömmerda bis zur Mündung. - Jb. Hall. Verb. 3, 89-124, Halle 1922.

Lehmann, H. \& R.: Die diluvialen Flußterrassen in der Umgebung von Halle/S. - Leopoldina 6, WaLtheR-Festschrift, 233-251, Leipzig 1930.

Ruske, R.: Gliederung des Pleistozäns im Geiseltal und in seiner Umgebung. - Geologie 10, 15,2168, Berlin 1961.

Ruske, R. \& M. WüNSCHE: Lösse und fossile Böden im mittleren Saale- und unteren Unstruttal. Geologie 10, 9-29, Berlin 1961.

Ruske, R.: Das Pleistozän zwischen Halle (Saale), Bernburg und Dessau. - Diss. math.-nat. Fak. Universität Halle 1962. - - Zur Entstehung des Gewässernetzes in der Umgebung von Halle/S. - Hercynia 1, 1963. - - Das Pleistozän zwischen Halle (Saale), Bernburg und Dessau. - Geologie 13, 570-597, Berlin 1964.

Ruske, R. \& M. Wünsche: Zur Gliederung des Pleistozäns im Raum der unteren Unstrut. - Geologie 13, 211-222, Berlin 1964.

Schulz, W.: Sedimentpetrographische Untersuchungen im Pleistozän westlich von Halle (Saale). Geologie 10,30-49, Berlin 1961. - - Gliederung des Pleistozäns in der Umgebung von Halle. - Geologie 11, Beiheft 36, Berlin 1962.

Siegert, L. \& W. Weissermel: Das Diluvium zwischen Halle und Weißenfels. - Abh. preuß. geol. Landesanst., N.F., H. 60, Berlin 1911.

ToEpFER, V.: Die glazialen und präglazialen Schotterterrassen im mittleren Saale-Tal und ihre Stellung in der geologischen und astronomischen Gliederung des Eiszeitalters. - Ber. Naturforsch. Ges. Freiburg i. Br. 32, 15-110, Freiburg i. Br. 1933.

Manuskr. eingeg. 23. 5. 1965.

Anschrift des Verf.: Dr. R. Ruske, 40 Halle/S., Angerstraße 1, VEB Geologische Erkundung West. 